\title{
Exploiting Structure Periodicity and Symmetry in Capacitance Calculations for Three-Dimensional Multiconductor Systems
}

\author{
RUEY-BEEI WU AND LEON L. WU
}

\begin{abstract}
The structure periodicity and symmetry usually encountered in the design and packaging of integrated circuits are utilized to dramatically alleviate the computation cost in the capacitance calculations for three-dimensional multiconductor systems by the integral equation method. For periodic structures, the region of unknowns is reduced to the base period by employing a modified Green's function which circumvents the periodicity singularity. For the structures with $s$ orthogonal planes of symmetry, where $s=1,2$, or 3 , the region of unknowns is reduced to $1 / 2^{s}$ of the original whole space by the help of even- and odd-mode decomposition techniques. Both algorithms are embedded into a general three-dimensional capacitance calculation program by which a numerical calculation for the via capacitance in a multilayer ceramic environment is presented and compared with the experimental measurements.
\end{abstract}

\section{INTRODUCTION}

T HE ADVENT OF smaller and denser integrated circuits and packages always leads to very large and complex systems with extremely small physical dimensions. The stray and interconnection capacitances which once were insignificant in large discrete components now become crucial elements in the modeling and computeraided electric analysis for high-performance systems [1].

Several methods have been proposed to deal with the capacitance calculations for three-dimensional multiconductor systems, such as the finite element method (FEM) [2] and the integral equation method (IEM) [3]-[7]. Both methods employ a large number of unknowns to approximate, for FEM, the potential distribution in the space surrounding the conductors or, for IEM, the charge distribution on the conductor surfaces. In other words, FEM models the three-dimensional space while IEM models the two-dimensional surfaces. FEM is advantageous for cases where the space is in a greatly varying or complex inhomogeneous environment. However, for most practical structures where the environment is homogeneous or at most has layered inhomogeneity, IEM is much more efficient and thus will be considered here.

Together with a suitable discretization procedure, IEM leads to the numerical solution of a matrix equation for which the required storage and computation time is pro-

Manuscript received August 31, 1987; revised April 18, 1988

R.-B. Wu is with the Department of Electrical Engineering, National Taiwan University, Taipei, Taiwan, R.O.C.

L. L. Wu is with the General Technology Division, East Fishkill Facility, IBM, Hopewell Junction, NY 12533.

IEEE Log Number 8822323 portional to the square and cubic of the number of unknowns, respectively. The number of unknowns which represent the two-dimensional surface charge increases very rapidly as the geometry becomes more complicated. Hence, attempts of IEM for practical structures are usually abandoned because either the amount of storage required is simply not available on any machine or the computation time required cannot be afforded.

Fortunately, the high level of integration in the present technology usually exhibits symmetry and periodicity in the integrated circuits and packages. By taking full advantage of structure periodicity and symmetry in these cases, the methods proposed here can considerably reduce the required computer storage and computation time without sacrificing numerical accuracy. With the reduction of computation time and especially storage, it becomes possible to analyze more complicated integrated circuits and packages.

For periodic structures, the most straightforward but time consuming method takes several periods into consideration and neglects the effects from farther periods. After solving the charge distribution in these periods, the perperiod capacitance can be found from the solution of the central period which is least influenced by the artificially chosen ends. However, recognizing that the charge distribution in the whole structure is periodic, a better method sums up the contributions from all the periods and only calls for the central base period [8]. Since this summation tends to infinity, the method includes only a finite number of summation terms by truncating the effects from farther periods but still worries if the number is large enough to ensure a convergent result. In this paper, a novel approach is proposed in Section II to circumvent this periodicity singularity. The effects from all the periods can be properly included and the results will be more accurate.

For symmetric structures, a purely mathematical approach has been proposed to reduce the computation required in the matrix inversion [9]. However, the simplification calls for the whole original matrix and the operation is rather complicated. A better modal decomposition technique is thus proposed in Section III, which gives an equivalent and more physical insight. This technique operates in a condensed matrix directly so that the operation is simpler and the required memory storage is smaller. 
Section IV describes the basic procedure employed in the numerical analysis and some computation considerations taken in the program setup. Several examples are then included in Section $\mathrm{V}$ to demonstrate and verify the significant features of this program. Also, the program is applied to analyze the via capacitance in a practical packaging structure and the results are compared with experimental measurements. Finally, some brief discussions and conclusions are presented in Section VI.

\section{Capacitances in Periodic Structures}

The integral equation method employed in the capacitance calculations for three-dimensional multiconductor systems can be formulated as follows. Let the structure consist of $M$ conductors, which are denoted $S_{m}(m=$ $1,2, \cdots, M)$. Similarly, let the imposed voltage and stored total charge on the $m$ th conductor be $V_{m}$ and $Q_{m}$, respectively. Then the charge density $\rho(\boldsymbol{r})$ on conductor surfaces satisfies the integral equation

$$
\begin{aligned}
& \sum_{m=1}^{M} \int_{S_{m}} G\left(\boldsymbol{r}, \boldsymbol{r}^{\prime}\right) \cdot \rho\left(\boldsymbol{r}^{\prime}\right) d \boldsymbol{r}^{\prime}=V(\boldsymbol{r})=V_{n} \\
& \text { for } \boldsymbol{r} \in S_{n}, \quad n=1,2, \cdots, M .
\end{aligned}
$$

here, the Green's function $G\left(\boldsymbol{r}, \boldsymbol{r}^{\prime}\right)=1 / 4 \pi \epsilon\left|\boldsymbol{r}-\boldsymbol{r}^{\prime}\right|$ when all these conductors are in a homogeneous medium of dielectric constant $\epsilon$. For structures with several layered dielectric regions, the Green's function is much more complicated [4]. Nevertheless, the simplifications proposed in this paper can be applied similarly.

By a suitable discretization procedure and the solution of a matrix equation [3]-[7], the unknown charge density and thus the total charge induced on each conductor can be approximately evaluated. Mathematically, the total charges are related to the voltages by

$$
\begin{aligned}
Q_{m}=\int_{S_{m}} \rho(\boldsymbol{r}) d \boldsymbol{r}= & \sum_{n=1}^{M} C(m, n) \cdot V_{n} \\
& \text { for } m=1,2, \cdots, M .
\end{aligned}
$$

Here, the coefficient $C(m, n)$, called the short-circuit capacitance [5], is the total charge induced on the $m$ th conductor when the $n$th conductor has a unit voltage imposed while all the others are grounded.

An equivalent but more common circuit capacitance, called the direct capacitance [3] or two-terminal capacitance [5], can be obtained from the short-circuit capacitance by

$$
C_{d}(m, m)=\sum_{n=1}^{M} C(m, n)
$$

and

$$
C_{d}(m, n)=-C(m, n) \quad \text { for } m \neq n .
$$

Here, the diagonal term $C_{d}(m, m)$ represents the selfcapacitance of the $m$ th conductor to the ground at infinity; while the off-diagonal term $C_{d}(m, n)$ represents the mutual capacitance between the two conductors $m$ and $n$.

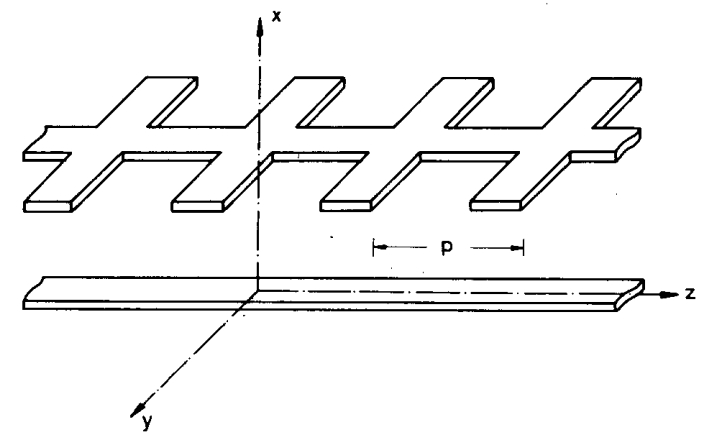

Fig. 1. A typical structure periodic in the $z$ direction with a period $p$.

Now, let us consider a typical periodical structure as shown in Fig. 1. Without loss of generality, the structure is assumed to be periodic along the $z$ axis with a period of $p$. When the conductors in different periods have the same voltages imposed, the charge density distribution in the whole structure is periodic, i.e., $\rho(\boldsymbol{r}+p \hat{z})=\rho(\boldsymbol{r})$. Therefore, the suitable Green's function in (1) should include the contributions from all the periods, i.e.,

$$
G_{p}\left(\boldsymbol{r}, \boldsymbol{r}^{\prime}\right)=\sum_{k=-\infty}^{\infty} G\left(\boldsymbol{r}, \boldsymbol{r}^{\prime}+k p \hat{z}\right)
$$

while only the unknown charge density in the central base $(k=0)$ period is required to be solved.

However, the Green's function $G_{p}$ is improper since the term $G\left(\boldsymbol{r}, \boldsymbol{r}^{\prime}+k p \hat{z}\right)$ tends to $(4 \pi \epsilon p|k|)^{-1}$ for large $|k|$, which makes the series in (4) divergent. Hence, it is advantageous to define instead a modified Green's function:

$$
\begin{aligned}
& \tilde{G}_{p}\left(\boldsymbol{r}, \boldsymbol{r}^{\prime}\right)=G\left(\boldsymbol{r}, \boldsymbol{r}^{\prime}\right) \\
& \quad+\sum_{k=1}^{\infty}\left[G\left(\boldsymbol{r}, \boldsymbol{r}^{\prime}+k p \hat{z}\right)+G\left(\boldsymbol{r}, \boldsymbol{r}^{\prime}-k p \hat{z}\right)-\frac{1}{2 \pi \epsilon p k}\right] .
\end{aligned}
$$

Now, the series (5) is convergent since the bracket in (5) is proportional to $k^{-3}$ for large $k$.

Substituting (5) into (1) gives

$$
\sum_{m=1}^{M} \int_{S_{m}} \tilde{G}_{p}\left(\boldsymbol{r}, \boldsymbol{r}^{\prime}\right) \cdot \rho\left(\boldsymbol{r}^{\prime}\right) d \boldsymbol{r}^{\prime}=V(\boldsymbol{r})-\alpha
$$

where the constant

$$
\alpha=\Sigma \cdot\left(\sum_{m=1}^{M} \int_{S_{m}} \rho\left(\boldsymbol{r}^{\prime}\right) d \boldsymbol{r}^{\prime}\right)=\Sigma \cdot\left(\sum_{m=1}^{M} Q_{m}\right)
$$

and

$$
\Sigma=\sum_{k=1}^{\infty}(2 \pi \epsilon p k)^{-1}
$$

It should be noted that $\Sigma$ is improper since the series is divergent. This in turn makes $\alpha$ and thus $\rho\left(\boldsymbol{r}^{\prime}\right)$ in (6) infinite unless the sum of total charges

$$
\sum_{m=1}^{M} \int_{S_{m}} \rho(\boldsymbol{r}) d \boldsymbol{r}=\sum_{m=1}^{M} Q_{m}=0 .
$$


In other words, for periodic structures, there is an additional charge constraint (7), i.e., the sum of total charges inside one period must be zero. Under this constraint, the constant $\alpha$ can be finite, but not necessarily zero.

The conventional capacitance calculation procedure can be applied similarly except that the modified Green's function $\tilde{G}_{p}$ should be employed while the total charge is expressed as

$$
Q_{m}=\sum_{n=1}^{M} \tilde{C}(m, n) \cdot\left(V_{n}-\alpha\right)
$$

where $\tilde{C}(m, n)$ is the modified capacitance. To find the proper capacitance $C(m, n)$, the parameter $\alpha$ must be solved. By the constraint (7), it is found that

$$
\alpha=\sum_{n=1}^{M}\left(D_{n} / \Delta\right) \cdot V_{n}
$$

where

$$
D_{n}=\sum_{m=1}^{M} \tilde{C}(m, n)
$$

and

$$
\Delta=\sum_{n=1}^{M} \sum_{m=1}^{M} \tilde{C}(m, n)=\sum_{n=1}^{M} D_{n} .
$$

Substituting (9) into (8), the short-circuit capacitance per period is

$$
C(m, n)=\tilde{C}(m, n)-\left(D_{m} \cdot D_{n}\right) / \Delta .
$$

Similarly, the two-terminal capacitance $C_{d}(m, n)$ per period can be obtained from $C(m, n)$ by (3). It is noted that the diagonal term $C_{d}(m, m)$ is always zero for periodic structures.

Since the summation term in the bracket of (5) has a $1 / k^{3}$ dependence for large $k$, the series $\tilde{G}_{p}$ converges but still slowly. A rough estimate shows that about 100 terms are required to converge the result into a relative error of $10^{-4}$. To speed up the rate of convergence, the summation term in the bracket of (5) can be expressed as

$$
\begin{aligned}
\tilde{G}_{k}\left(\boldsymbol{r}, \boldsymbol{r}^{\prime}\right) & =G\left(\boldsymbol{r}, \boldsymbol{r}^{\prime}+k p \hat{z}\right)+G\left(\boldsymbol{r}, \boldsymbol{r}^{\prime}-k p \hat{z}\right)-(2 \pi \epsilon k p)^{-1} \\
= & \left\{3\left[\left(\boldsymbol{r}-\boldsymbol{r}^{\prime}\right) \cdot \hat{z}\right]^{2}-\left|\boldsymbol{r}-\boldsymbol{r}^{\prime}\right|^{2}\right\} / 4 \pi \epsilon k^{3} p^{3}+R .
\end{aligned}
$$

Here, the remainder $R$ converges much faster since it has a $1 / k^{5}$ dependence for large $k$. The series of the first term on the right-hand side of (11), which, however, is slowly convergent, can be evaluated exactly since

$$
\sum_{k=1}^{\infty} \frac{1}{k^{3}}=\zeta_{3}=1.2020563 \cdots \text {. }
$$

By this simplification, it is noted that the same convergence error of $10^{-4}$ can usually be achieved in about ten terms.

\section{Capacitances in Symmetric Structures}

The even- and odd-mode decomposition techniques are generalized in this section to ease the capacitance calculations for multiconductor structures which contain $s(s=1$,

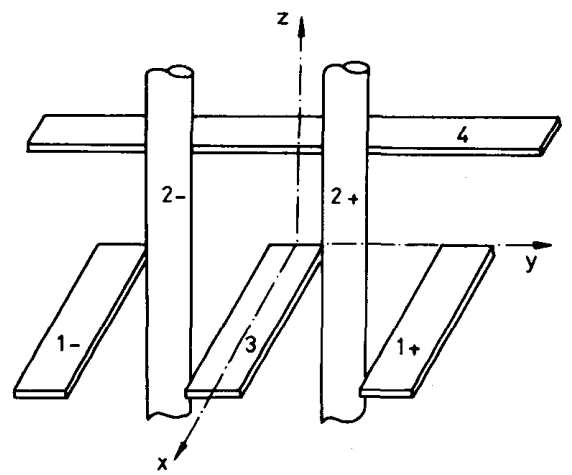

Fig. 2. A typical structure symmetric with respect to the $x=0$ and $y=0$ planes. Here, conductors 3 and 4 are cross-symmetric, while conductors 1 and 2 are open-symmetric.

2 , or 3) orthogonal planes of symmetry. First, consider the simplest case, where the structure has one plane of symmetry, say the $y=0$ plane. As shown in Fig. 2, there are four conductors in the region $y \geqslant 0$. The conductors which are shorted with their image counterparts in the $y \leqslant 0$ region are called cross-symmetric conductors, e.g., conductors 3 and 4 in Fig. 2. Those which are separate from their image counterparts, e.g., conductors 1 and 2 in Fig. 2, are called open-symmetric conductors.

To be more general, assume there are $K$ conductors in the region $y \geqslant 0$. The open-symmetric conductors are numbered as the first $L$ conductors, denoted as conductor $i_{+}$in the $y>0$ region and conductor $i_{-}$in the $y<0$ region $(i=1,2, \cdots, L)$. While the cross-symmetric conductors are numbered as the $(L+1)$ st, $(L+2)$ nd, $\cdots, K$ th conductors. In fact, there are $L+K$ conductors in the whole space. However, by means of the modal decomposition technique, only the capacitances between the $K$ conductors in $y \geqslant 0$ region need be solved to find the capacitances between all these $K+L$ conductors.

To facilitate the analysis, let us define three vectors for the total charges, i.e., $\boldsymbol{Q}_{+}-\left[Q_{1_{1}}, Q_{2}, \cdots, Q_{L_{+}}\right]$and $\boldsymbol{Q}_{-}=$ $\left[Q_{1}, Q_{2}, \cdots, Q_{L_{-}}\right]$for the open-symmetric conductors in the $y>0$ and $y<0$ regions, respectively; in addition, $\boldsymbol{Q}_{c}=\left[Q_{L+1}, Q_{L+2}, \cdots, Q_{K}\right]$ for the cross-symmetric conductors. Accordingly, the voltages imposed on all the conductors are grouped into three voltage vectors: $\boldsymbol{V}_{+}, \boldsymbol{V}_{-}$, and $\boldsymbol{V}_{\boldsymbol{c}}$. Then, the desired short-circuit capacitance matrix can be written as

$$
\left[\begin{array}{l}
\boldsymbol{Q}_{+} \\
\boldsymbol{Q}_{c} \\
\boldsymbol{Q}_{-}
\end{array}\right]=\left[\begin{array}{ccc}
\overline{\bar{C}}_{++} & \overline{\bar{C}}_{+c} & \overline{\bar{C}}_{+-} \\
\overline{\bar{C}}_{c+} & \overline{\bar{C}}_{c c} & \overline{\bar{C}}_{c-} \\
\overline{\bar{C}}_{-+} & \overline{\bar{C}}_{-c} & \overline{\bar{C}}_{--}
\end{array}\right] \cdot\left[\begin{array}{c}
\boldsymbol{V}_{+} \\
\boldsymbol{V}_{c} \\
\boldsymbol{V}_{-}
\end{array}\right]
$$

where, due to symmetry, the partitioned capacitance matrices should satisfy $\overline{\bar{C}}_{++}=\overline{\bar{C}}_{--}, \overline{\bar{C}}_{+c}=\overline{\bar{C}}_{-c}=\overline{\bar{C}}_{c+}=$ $\overline{\bar{C}}_{c-}$, and $\overline{\bar{C}}_{+-}=\overline{\bar{C}}_{-+}$.

When the imposed voltage distribution is even-symmetric, i.e., $\boldsymbol{V}_{+}=\boldsymbol{V}_{-}$, the charge distribution is even-symmet- 
ric also, i.e., $\boldsymbol{Q}_{+}=\boldsymbol{Q}_{-}$. Only the unknown charge density in the $y \geqslant 0$ region is necessary since the charge density on the image counterparts in the $y \leqslant 0$ region is the same. The governing integral equation is thus similar to (1) except that the Green's function $G\left(\boldsymbol{r}, \boldsymbol{r}^{\prime}\right)$ is replaced by

$$
G_{e}\left(\boldsymbol{r}, \boldsymbol{r}^{\prime}\right)=G\left(\boldsymbol{r}, \boldsymbol{r}^{\prime}\right)+G\left(\boldsymbol{r}, \underline{\boldsymbol{r}}^{\prime}\right)
$$

where $\boldsymbol{r}^{\prime}$ is the image of $\boldsymbol{r}^{\prime}$ with respect to the plane of symmetry. For example, $\underline{\boldsymbol{r}}^{\prime}=(x,-y, z)$ when $\boldsymbol{r}^{\prime}=(x, y, z)$ and the $y=0$ plane is the plane of symmetry. Note that only the structure in the $y \geqslant 0$ region is required; i.e., both $\boldsymbol{r}$ and $\boldsymbol{r}^{\prime}$ are in the $y \geqslant 0$ region.

The charge density in the $y \geqslant 0$ region and thus the short-circuit capacitance matrix can be obtained following a similar solution procedure in deriving (2). Since the charge and voltage distributions are even-symmetric in this case, the matrix can be called the even-mode short-circuit capacitance matrix. By the relations that $\boldsymbol{V}_{+}=\boldsymbol{V}_{-}$and $Q_{+}=Q_{-}$, this matrix can also be expressed from (12) as

$$
\left[\begin{array}{c}
\boldsymbol{Q}_{+} \\
\boldsymbol{Q}_{c} / 2
\end{array}\right]=\left[\begin{array}{cc}
\overline{\bar{C}}_{++}+\overline{\bar{C}}_{+-} & \overline{\bar{C}}_{+c} \\
\overline{\bar{C}}_{+c} & \overline{\bar{C}}_{c c} / 2
\end{array}\right] \cdot\left[\begin{array}{c}
\boldsymbol{V}_{+} \\
\boldsymbol{V}_{c}
\end{array}\right]=\left[\overline{\bar{C}}_{e}\right] \cdot\left[\begin{array}{c}
\boldsymbol{V}_{+} \\
\boldsymbol{V}_{c}
\end{array}\right]
$$

Here, the vector $\boldsymbol{Q}_{c}$ is divided by two since the charge in the $y \geqslant 0$ region is half of that in the whole region for cross-symmetric conductors.

Similarly, each conductor and its image counterpart have opposite charge distributions, i.e., $\boldsymbol{Q}_{-}=-\boldsymbol{Q}_{+}$, when the imposed voltages are opposite, i.e., $\boldsymbol{V}_{-}=-\boldsymbol{V}_{+}$. Here, the plane of symmetry is equivalent to a grounded plane, which implies that all the cross-symmetric conductors are grounded, i.e., $\boldsymbol{V}_{c}=\mathbf{0}$. The solution approach is the same as that for the even-symmetric case except that the Green's function now becomes

$$
G_{o}\left(\boldsymbol{r}, \boldsymbol{r}^{\prime}\right)=G\left(\boldsymbol{r}, \boldsymbol{r}^{\prime}\right)-G\left(\boldsymbol{r}, \underline{\boldsymbol{r}}^{\prime}\right) .
$$

Here, the subscript $o$ stands for the odd-symmetric case, in contrast to the subscript $e$ in (13) for the even-symmetric case.

The resultant matrix is now called the odd-mode shortcircuit capacitance matrix, which can be expressed as

$$
\left(\boldsymbol{Q}_{+}\right)=\left(\overline{\bar{C}}_{++}-\overline{\bar{C}}_{+-}\right) \cdot\left(\boldsymbol{V}_{+}\right)=\left(\overline{\bar{C}}_{o}\right) \cdot\left(\boldsymbol{V}_{+}\right) .
$$

Here, the relations $V_{-}=-V_{+}, Q_{-}=-Q_{+}$, and $V_{c}=0$ are substituted into (12).

By choosing the suitable Green's functions (13) and (15), only the unknown charge density in the $y \geqslant 0$ region is required to obtain (14) and (16), respectively. However, the whole short-circuit capacitance matrix in (12) can be achieved accordingly. Let $C_{e}(i, j)$ and $C_{o}(i, j)$ be the even- and odd-mode capacitances between two conductors $i$ and $j$ in the $y \geqslant 0$ region. The equivalent capacitances in the whole space can be solved as follows. When both conductors $i$ and $j$ are cross-symmetric,

$$
C(i, j)=2 C_{e}(i, j) \text {. }
$$

When one conductor, say the $i$ th, is cross-symmetric while the other, say the $j$ th, is open-symmetric,

$$
C\left(i, j_{+}\right)=C\left(i, j_{-}\right)=C_{e}(i, j) .
$$

When both conductors $i$ and $j$ are open-symmetric,

$$
\begin{aligned}
& C\left(i_{+}, j_{+}\right)=C\left(i_{-}, j_{-}\right)=\left[C_{e}(i, j)+C_{o}(i, j)\right] / 2 \\
& C\left(i_{-}, j_{+}\right)=C\left(i_{+}, j_{-}\right)=\left[C_{e}(i, j)-C_{o}(i, j)\right] / 2 .
\end{aligned}
$$

This approach can be extended for the structures with two or three orthogonal planes of symmetry. The basic algorithm can be briefly described by a simple structure with two orthogonal planes of symmetry, say, the $x=0$ and $y=0$ planes. By first assuming that the $x=0$ plane is even-symmetric, the structure of interest is in the half-space with $x \geqslant 0$. Now, the $y=0$ plane is the plane of symmetry in the "new" half-space. The aforementioned modal decomposition technique can be applied similarly except that the Green's function $G\left(\boldsymbol{r}, \boldsymbol{r}^{\prime}\right)$ should be substituted by the Green's function in the "new" half-space, i.e., $G\left(x, y, z ; x^{\prime}, y^{\prime}, z^{\prime}\right)+G\left(x, y, z ;-x^{\prime}, y^{\prime}, z^{\prime}\right)$. By solving the unknown charge density for the two cases where the $y=0$ plane is even- and odd-symmetric, the resultant short-circuit capacitance matrix in the "new" half-space can be obtained by employing relation (17). This matrix is now the even-mode capacitance matrix $\overline{\bar{C}}$ with respect to the $x=0$ plane in the original whole space.

Similarly, the odd-mode capacitance matrix $\overline{\bar{C}}_{o}$ can be obtained by first assuming that the $x=0$ plane is oddsymmetric. By applying (17) again, the desired short-circuit capacitance matrix can be obtained from $\overline{\bar{C}}_{e}$ and $\overline{\bar{C}}_{o}$. It is noted that this approach requires only the charge density in the $x \geqslant 0$ and $y \geqslant 0$ region, which means one quarter of the original total unknowns. However, four cases must be solved, corresponding to the assumptions that each of the $x=0$ and $y=0$ planes is either even- or odd-symmetric.

Roughly speaking, the numerical solution for a matrix equation of $N$ unknowns calls for $N^{2} / 2$ memory storage. The computation time, if taking into account the computation of matrix elements, is proportional to $N^{\gamma}(2<\gamma<3)$. For cases with small $N, \gamma \simeq 2$ since the computation of matrix elements dominates; while $\gamma=3$ for cases with large $N$ whence the solution of matrix equation dominates. Hence this approach, when applied to structures with two orthogonal planes of symmetry, reduces the number of unknowns by a factor of 4 , and thus the memory storage by 16 . Since it is usually necessary to solve four cases, corresponding to different assumptions on the $x=0$ and $y=0$ planes, the computation time to obtain the final $C(m, n)$ in the whole space is reduced by a factor of $(16-64) \div 4=4-16$.

For structures with three orthogonal planes of symmetry, the solution approach is similar. The reduction in computation cost, however, is tremendous, i.e., 64 times in memory storage and 8-64 times in computation time.

\section{SOlution APPROACH}

Regardless of whether the structure is periodic, symmetric, or neither, the capacitance calculation requires a solution of the unknown surface charge density from the 
integral equation like (1), and then a relation in terms of short-circuit capacitances like (2). The basic procedure and some related numerical considerations are briefly described as follows. First, each conductor is divided into small cells. The surface charge density may be different from one cell to another but is assumed to be a constant inside the same cell. Theoretically, a better solution will be achieved by a finer discretization. However, it is always accompanied by an enormous increase in the number of unknowns and thus the computation cost. A good algorithm which can achieve reasonable accuracy with the least discretization is desired.

Let the surface of the $m$ th conductor, $S_{m}$, be partitioned into $N_{m}$ cells, i.e., $S_{m_{j}}\left(j=1,2, \cdots, N_{m}\right)$. Denoting by $q_{m}$ the unknown total charge on the cell $S_{m_{j}}$, the integral equation (1) becomes

$$
\sum_{m=1}^{M} \sum_{j=1}^{N_{m}}\left(\frac{1}{\left|S_{m_{j}}\right|} \int_{S_{m_{j}}} G\left(\boldsymbol{r}, \boldsymbol{r}^{\prime}\right) d \boldsymbol{r}^{\prime}\right) q_{m_{j}}=V(\boldsymbol{r})=V_{n}
$$

where $\left|S_{m_{j}}\right|$ denotes the area of the cell $S_{m_{j}}$. It is noted that the Green's function here should be replaced by $\tilde{G}_{p}$ for periodic structures and by $G_{e}$ or $G_{o}$ for symmetric structures.

It is crucial to calculate the integral in (18) accurately and efficiently. The closed-form formula has been derived for the cells with rectangular [3] or triangular [6] shape. The triangular cell is more general in modeling arbitrary geometry, but the involved computation is more time-consuming. For practical packaging structures, rectangular cells suit as well [4], [5] and are employed here.

Two methods have been proposed to solve the unknowns $q_{m}$ in (18). The simpler, collocation method [3], [6] matches (18) at the center of each cell, while the Galerkin method [4] matches the average of (18) inside the same cell. It can be shown that the Galerkin method achieves a variational solution [10]; i.e., a first-order error of charge distribution results in a much smaller secondorder error of resultant capacitances. Hence, though with a little extra computation complexity, the Galerkin method usually achieves much better solutions.

Applying Galerkin's method to the integral in (18) cell by cell, we can achieve a system of simultaneous linear equations for unknown charges $q_{m}$, i.e.,

$$
\begin{aligned}
& \sum_{m=1}^{M} \sum_{j=1}^{N_{m}} P_{n_{i} m_{j}} \cdot q_{m_{j}}=V_{n}, \\
& \quad i=1,2, \cdots, N_{n} ; n=1,2, \cdots, M
\end{aligned}
$$

where

$$
P_{n_{i} m_{j}}=\frac{1}{\left|S_{n_{i}}\right| \cdot\left|S_{m_{j}}\right|} \int_{S_{n_{i}}} \int_{S_{m_{j}}} G\left(\boldsymbol{r}, \boldsymbol{r}^{\prime}\right) d \boldsymbol{r} d \boldsymbol{r}^{\prime} .
$$

It is noted by inspection that the coefficient matrix composed of $P_{n_{i} m_{j}}$ is symmetric, which makes the solution of matrix equation (19) easier.

A closed-form formula for the coefficient $P_{n_{i} m_{j}}$ can be derived when the two rectangular cells $S_{n_{i}}$ and $S_{m_{j}}$ are either parallel or orthogonal [4]. It may happen in practical structures that the orientation between some cells is neither parallel nor orthogonal. For example, the two cells along the periphery of a circular cylinder always have more general orientations. In those cases, the inner integral is evaluated by the closed-form expression while the outer integral is approximated by the Gaussian quadrature. A higher order quadrature formula should be employed to ensure accuracy for very close cells. In most cases where the cells are not very close, a rough formula which requires less computation is sufficient.

A Fortran program is thus impiemented on the IBM/3090 mainframe computer to calculate the capacitances for three-dimensional multiconductor systems. To divide the conductor surfaces into rectangular cells, the program first requests a data input for the conductor geometries and the discretization sizes. Many complicated geometries can be partitioned into several rectangular basic boxes. It is thus easier to specify the geometries by defining the coordinates of the boxes and specifying that the surfaces joining two boxes are charge-free [5]. If a surface includes the edge, a nonuniform division should be chosen to achieve a good accuracy [4].

For periodic and/or symmetric structures, the data input for conductor geometry is greatly simplified since only part of the whole structure is actually required in solution. When the structure is periodic, the program requests only the part of geometry in one period of the structure instead of the whole geometry in many periods. For symmetric structures with $s$ planes of symmetry $(s=1,2$, or 3 ), only $1 / 2^{s}$ of the whole geometry is necessary.

The Galerkin method is then applied to obtain a matrix equation, which is solved by the Gaussian elimination method to find the unknown charge density and thus the capacitances as expressed in (2). If the system has periodicity or symmetry, the simplifications described in Section II or III should be imposed on these capacitances to obtain the desired capacitances.

\section{Numerical Examples}

To demonstrate the significant features of this program, several examples are presented in this section. The first example considers the average capacitance per centimeter in a coaxial transmission line where the two conductors have a radius ratio of two and the inside region is free space. This example is chosen because its solution is well known, enabling us to gain insight into the rate of convergence and the necessity of the proposed simplification for periodic structures.

To analyze this simple structure, one may assume that the cable is of finite length and employs the conventional three-dimensional capacitance calculation. Shown in Fig. 3 is the resultant average capacitance per centimeter versus the length of the coaxial cable. As the length increases, it tends to the exact two-dimensional capacitance, which, as shown by the dotted line in the figure, is $2 \pi \epsilon / \ln 2=$ $0.80262 \cdots \mathrm{pF} / \mathrm{cm}$. However, the rate of convergence is rather slow. The relative error is still greater than 3 per- 


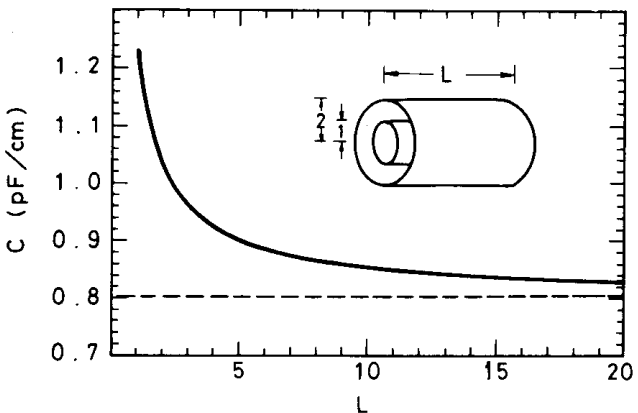

Fig. 3. Average capacitance per centimeter versus length for a coaxial cable. Dotted curve denotes the exact result as the length tends to infinity.

cent, even when the ratio of length to outer radius is as large as 10 .

It is noted that any two-dimensional structure is periodic along the third dimension with a period $p$, which can be assumed to have any positive value. Hence, one may also employ the three-dimensional method together with the periodicity simplification to handle two-dimensional capacitance calculations. By arbitrarily choosing a period, say $p=2$, the program obtains the result $0.80259 \mathrm{pF} / \mathrm{cm}$, which is very close to the exact solution. This at least validates the simplification algorithm proposed here for periodic structures.

As shown in Fig. 3, the discrepancy between the finitelength and the infinite-length results depends on the ratio of the longitudinal length to the transversal size. Since a two-dimensional transmission line can be considered a periodic structure with arbitrary period, the number of periods inside a fixed length being arbitrary. Therefore, it is very difficult for the truncation approach [8] to achieve a meaningful criterion for a number of periods which will be sufficient to ensure a convergent result. The modified Green's function together with the associated algorithm proposed here includes the effects from all the periods and thus can always achieve better results.

The second example considers a symmetric structure with eight cubic conductors of size $1 \mathrm{~cm}$ centered at $\left(x_{0}, y_{0}, z_{0}\right)$ in free space where $x_{0}, y_{0}$ and $z_{0}$ are either +1 or $-1 \mathrm{~cm}$. The surfaces of the conductors are parallel to the coordinate axes. Therefore, the structure has three planes of symmetry. By exploring the structure symmetry, only the structure in one eighth of the whole space, i.e., the conductor centered at $(1,1,1)$, is required in the solution. When all three planes of symmetry are assumed even-symmetric, the resultant even-mode capacitance is $0.2645 \mathrm{pF}$. Similarly, when two, one, or none of the three planes are even-symmetric, the capacitances are, respectively, 0.8647 , 1.201 , and $1.417 \mathrm{pF}$. By relations (17) and (3), the twoterminal capacitances between all eight conductors can be obtained. The self-capacitance is $0.2645 \mathrm{pF}$, while the mutual capacitances between adjacent, face-diagonal, and cube-diagonal conductors are $0.1860,0.04804$, and 0.01790 $\mathrm{pF}$, respectively. Here, each side of the conductor is divided into four segments; i.e., the number of unknowns is

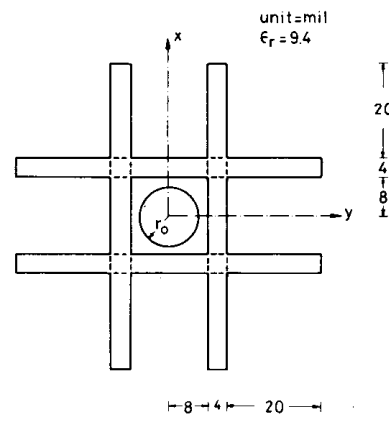

(a)

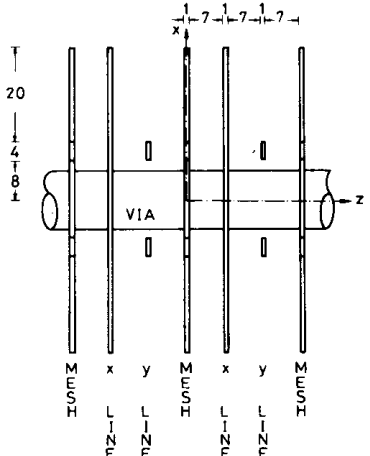

(b)
Fig. 4. A practical packaging structure consisting of via conductor, mesh planes, and $x$ and $y$ lines. (a) Top view. (b) Side view.

$6 \times 4 \times 4=96$. The computation time required to find the final solution is about 7 seconds.

On the other hand, the same program is also employed to solve the original structure without utilizing the structure symmetry. Keeping the same discretization size, the two-terminal capacitances between all eight conductors are exactly the same as those obtained before. This again validates the suitability of the proposed simplification algorithm for symmetric structures. However, the number of unknowns is 768 while the computation time is $144 \mathrm{sec}-$ onds now. This means that exploiting structure symmetry here can result in a reduction in memory storage and computation time by 64 and 20 times, respectively.

Finally, consider a practical packaging structure made of multilayer ceramic for high-performance computers [11]. The structure includes many perforated mesh planes inside which the $x$ - and $y$-directed lines are designed for the signal transmission between integrated-circuit chips. In addition, many via conductors are built through the holes in mesh planes to serve as the $z$-directed signal or power connection between different layers.

For practical computer systems where the rise time of the signal is about $1 \mathrm{~ns}$, the structure size is much smaller than the signal wavelength of interest. Some characteristics, e.g., the impedance of a via conductor and the loading effect of the $x$ and $y$ lines, are reflected from the average capacitance between the via conductor and the grounded mesh planes. To analyze this problem, we consider a simplified structure as shown in Fig. 4 where (a) is the top view and (b) is the side view. The structure is assumed to be periodic in the $z$ direction but truncated in the transverse direction. The mesh planes and $x$ and $y$ lines are shorted together and considered as the ground. The relative dielectric constant of the surrounding material is 9.4 .

Since the structure is periodic in the $z$ direction as well as symmetric with respect to both the $x=0$ and $y=0$ planes, we only need to solve the one-period structure in the $x \geqslant 0$ and $y \geqslant 0$ region, which is modeled by 256 cells as shown in Fig. 5. The via capacitance can now be obtained directly from the even-mode capacitance since 


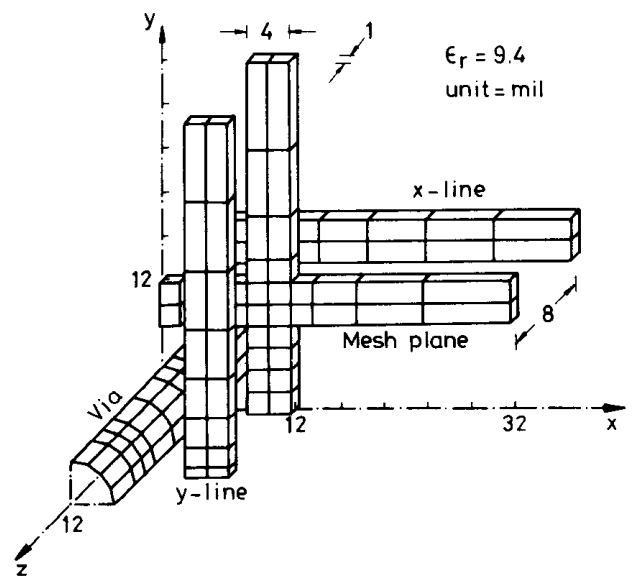

Fig. 5. Cells employed to model the structure (see Fig. 4) inside one period and in the $x \geqslant 0, y \geqslant 0$ region.

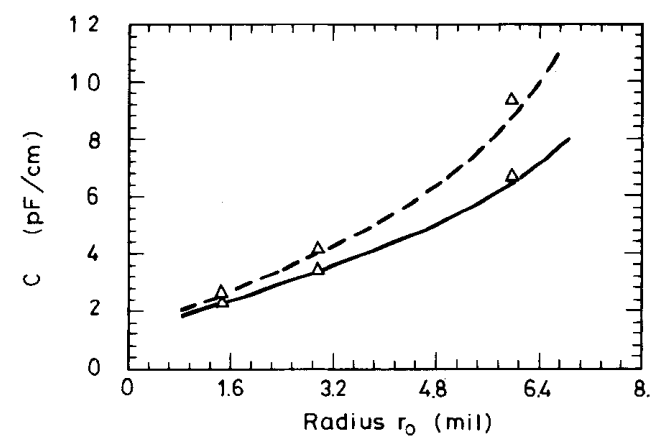

Fig. 6. Average capacitance between via conductor and mesh plane versus via conductor radius. Solid and dotted curves correspond to the cases where the $x$ and $y$ lines are absent and present, respectively. Triangular marks denote the measurement results.

both the conductors are cross-symmetric. The via capacitance versus the via conductor radius is shown by the dotted curve in Fig. 6, for which the computation time is about 44 seconds for a radius. As the radius increases, the distance between via conductor and mesh planes decreases and thus the capacitance increases significantly.

It is well known that the capacitance per unit length remains invariant when the structure is scaled by a constant factor. Based on this idea, a $150 \times$ scaled test vehicle including $M$ periods $(M \leqslant 7)$ was constructed to measure the capacitance of a via conductor with respect to the grounded mesh planes and $x$ and $y$ lines. To cancel the edge effect from both ends due to finite $M$ periods, two measurements with different $M$ 's, say $M=7$ and $M=5$, are executed and subtracted to give the average capacitance per unit length [12]. The results are shown by the triangular marks in the figure. It is found that the computational and experimental results compare favorably.

Now, consider the via capacitance without the loading effect of the $x$ and $y$ lines. When the $x$ and $y$ lines are absent, the structure is symmetric along the $z$ direction also. Then, only the half-period in the $z \geqslant 0$ region need be modeled. The number of unknowns is reduced to 92 and the computation time to 7 seconds. The resultant via capacitance is shown by the solid curve in Fig. 6, which is again consistent with the measurements. As compared with the case when the $x$ and $y$ lines are present, the via capacitance is reduced more significantly for larger via conductor radius. The relative reduction is 10 percent when the radius ratio is 0.1 , while it becomes 40 percent when the radius ratio is increased to 0.85 .

\section{Conclusions and Discussions}

A novel approach characterized by its capability to utilize structure periodicity and symmetry is proposed to deal with the capacitance calculations for three-dimensional multiconductor systems. The modified Green's function is proposed to circumvent the singularity due to infinite periods. In the meantime, it is shown that the sum of total charges in a periodic structure should be zero. By this approach, only the conductor geometry in one period of the structure need be modeled and solved while the effects from all the periods are included properly.

On the other hand, the idea of the modal decomposition technique is extended for multiconductor structures with several orthogonal planes of symmetry. Corresponding to each plane of symmetry, the structure is halved to find even- and odd-mode capacitances by assuming that the voltage and charge distributions in two half-spaces are even- and odd-symmetric, respectively. A general algorithm relating the original capacitances to the even- and odd-mode capacitances is derived for the multiconductor cases. For structures with several planes of symmetry, the procedure can be applied directly by repeating the algorithm several times. The geometry is easier to specify in data input and the computation cost is greatly reduced. In addition, fewer unknowns and thus fewer operations are involved, so that the roundoff error during numerical computation is reduced.

Combined with the two simplification algorithms for periodic and symmetric structures, the program is very general for analyzing the capacitances for practical packaging structures, such as the capacitance between two perforated mesh planes and the capacitance between two signal lines in the presence of mesh planes and crossing lines. However, it cannot handle structures with inhomogeneous material in its present form. In those cases, the material can be replaced with free space by placing unknown charges along the material boundary [7], but the simplification formula for periodic structures should be modified slightly.

It should be emphasized that in this paper the conductors in different periods for periodic structures are assumed shorted together; i.e., the voltage distribution is identical for all the periods. It is not uncommon for the conductors in different periods to be discrete and to have different voltages imposed. In those cases, it is sometimes important to calculate the capacitances between two conductors in different periods. A novel method to solve this difficulty is in progress and will be presented in the near future. 


\section{REFERENCES}

[1] A. E. Ruehli, "Survey of computer-aided electrical analysis of integrated circuit interconnection," IBM J. Res. Develop., vol. 23. pp. 626-639, Nov. 1979

[2] P. E. Cottrell and E. M. Buturla, "VLSI wiring capacitance," $I B M$ J. Res. Develop., vol. 29, pp. 277-288, May 1985.

[3] P. D. Patel, "Calculation of capacitance coefficients for a system of irregular finite conductors on a dielectric sheet," IEEE Trans. Microwave Theory Tech., vol. MTT-19, pp. 862-869, Nov. 1971.

[4] A. E. Ruehli and P. A. Brennan, "Efficient capacitance calculations for three-dimensional multiconductor system," IEEE Trans. Microwave Theory Tech., vol. MTT-21, pp. 76-82, Feb. 1973.

[5] A. E. Ruehli and P. A. Brennan, "Capacitance models for integrated circuit metallization wires," IEEE J. Solid-State Circuits, vol. SC-10, pp. 530-536, Dec. 1975

[6] S. M. Rao, A. W. Glisson, D. R. Wilton, and B. S. Vidula, "A simple numerical solution procedure for statics problems involving arbitrary-shaped surfaces," IEEE Trans. Antennas Propagat., vol. AP-27, pp. 604-608, Sept. 1979.

[7] S. M. Rao, T. K. Sarker, and R. F. Harrington, "The electrostatic field of conducting bodies in multiple dielectric media," IEEE Trans. Microwave Theory Tech., vol. MTT-32, pp. 1441-1448, Nov. 1984.

[8] H. C. Lee and H. D. Chai, "Integral point-matching method for two-dimensional Laplace field problems with periodical boundaries," IBM J. Res. Develop., vol. 24, pp. 622-630, Sept. 1980.

[9] W. T. Weeks, "Employing symmetry in electrical packaging analysis," IBM J. Res. Develop., vol. 23, pp. 669-674, Nov. 1979.

[10] R. F. Harrington, Field Computation by Moment Methods. New York: Macmillan, 1968.

[11] B. T. Clark and Y. M. Hill, "IBM multichip multilayer ceramic modules for LSI chips-Design for performance and density," IEEE Trans. Components, Hybrids, Manuf. Technol., vol. CHMT-3, pp. 89-93, Mar. 1980.

[12] Experiment conducted by D. C. Diehl in Yorktown Heights, IBM.

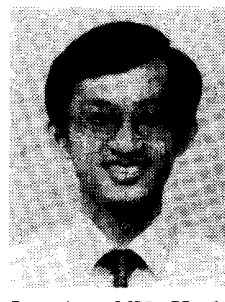

Ruey-Beei Wu was born in Tainan, Taiwan, Republic of China, on October 27, 1957. He received the B.S.E.E. degree from National Taiwan University, Taipei, Taiwan, in 1979, and the $\mathrm{Ph} . \mathrm{D}$. degree from the same university in 1985.

In 1982 , he joined the faculty of the Department of Electrical Engineering, National Taiwan University, where he is now an Associate Professor. In 1986, he was a Visiting Scientist for one year at the IBM General Technology Division Laboratory, East Fishkill Facility, Hopewell Junction, NY. He is involved in research on numerical technique for electromagnetics and electrical analysis for computer packaging.

$\mathbf{2}$

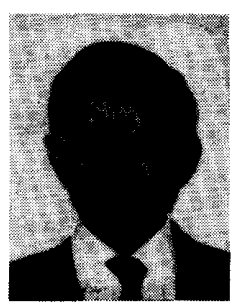

Leon $\mathbf{L}$. Wu received the B.S. degree in electrical engineering from National Taiwan University, Taipei, Taiwan in 1962 and the Ph.D. degree, also in electrical engineering, from Carnegie Mellon University, Pittsburgh, PA, in 1968.

After his graduate work he joined the IBM East Fishkill Laboratory, where he worked on silicon defect studies and on silicon device and process modeling. In 1977 , he started his work on packaging modeling. Since then, he has been involved in developing advanced packaging schemes for high-performance mainframe computers at IBM. 